\title{
Extranodal Malignant Lymphoma Originating from the Parapharyngeal Space: Two Cases
} \author{
Hideo Sakuma ${ }^{4}$ and Akiyoshi Konno ${ }^{2}$ \\ ${ }^{1}$ Department of Otorhinolaryngology/Head and Neck Surgery, Chiba University Graduate School of Medicine, Japan \\ ${ }^{2}$ Department of Otorhinolaryngology, Southern TOHOKU General Hospital, Japan \\ ${ }^{3}$ Department of Radiology, Southern TOHOKU General Hospital, Japan \\ ${ }^{4}$ Department of Pathological diagnosis, Southern TOHOKU General Hospital, Japan
}

Ichiro Fukumoto1*, Toyoyuki Hanazawa ${ }^{1}$, Yuji Ueki², Yusuke Mada ${ }^{2}$, Hiroyuki Matsuyama², Tatsuhiko Nakasato ${ }^{3}$,

Submission: March 23, 2021; Published: April 26, 2021

*Corresponding author: Ichiro Fukumoto, Department of Otorhinolaryngology/ Head and Neck Surgery, Chiba University Graduate School of Medicine, Japan

\section{Abstract}

Parapharyngeal space tumors develop deep inside the body and present with a wide variety of histological types, making them difficult to diagnose and treat. Here, we report two cases of lymphomas originating from the parapharyngeal space.

Case 1: A 47-year-old man complaining of right facial numbness and right ear pain was found to have a tumor extending from the right parapharyngeal space to the skull base on magnetic resonance imaging. He underwent a biopsy under general anesthesia and was diagnosed with diffuse large B cell lymphoma.

Case 2: A 60-year-old man presenting with left neck pain underwent a nasopharyngeal biopsy under general anesthesia for a tumor in the left parapharyngeal space, although a diagnosis could not be reached. After biopsy, the tumor enlarged dramatically and extended into the neck and skull base along the parapharyngeal space. A needle biopsy from below the left ear led to a diagnosis of diffuse large B cell lymphoma. Both patients underwent R-CHOP therapy and survived; their tumors were found to have disappeared on imaging investigations. Parapharyngeal space tumors, which extend diffusely along the parapharyngeal space to the neck and skull base, it is highly likely to be malignant lymphomas if the patient complains of intense pain.

Keywords: Parapharyngeal space tumor; Diffuse large B cell lymphoma; Extranodal malignant lymphoma; Histological transformation

\section{Introduction}

The parapharyngeal space lies lateral to the pharyngeal cavity and has an inverted pyramid shape extending vertically from the skull base to the greater cornu of the hyoid bone. The space is surrounded anteriorly, posteriorly, medially, and laterally by the medial pterygoid muscle, prevertebral fascia, pharyngeal constrictor muscles, and deep parotid fascia, respectively. Within the parapharyngeal space, there are major vessels and nerves, such as the internal jugular vein, internal carotid artery, sympathetic nerves, glossopharyngeal nerve, vagus nerve, and hypoglossal nerve. Anatomically, the parapharyngeal space is divided by the styloid process into an anterior pre-styloid compartment and a posterior post-styloid compartment; this division is important for differentiating parapharyngeal space tumors [1]. Primary parapharyngeal space tumors account for approximately $0.5 \%$ of all head and neck neoplasms [2].

Clinical symptoms of parapharyngeal space tumors do not manifest readily, causing frequent delays in diagnosis. In addition, the parapharyngeal space is anatomically located deep inside the body, hindering preoperative biopsy-based histological diagnosis. Consequently, the treatment of malignancies is often delayed. In nearly all cases, the first-line treatment is surgery, which can be performed using a variety of approaches, such as the transoral approach, transparotid approach, and transcervical approach. When an adequate visual field cannot be secured, midline mandibulotomy may be required [3]. Although surgery has recently been performed with endoscopes and robots [4,5], these 


\section{Global Journal of Otolaryngology}

surgeries often involve a high degree of difficulty. In this case report, we describe two cases of relatively rare parapharyngeal space tumors, which were difficult to diagnose, along with a discussion of the relevant literature.

\section{Case Presentation}

\section{Case 1}

A 47-year-old man presented with complaints of right facial numbness and right ear pain. Cranial computed tomography (CT) by a local neurologist did not reveal any abnormalities. Six months later, he visited his previous otorhinolaryngologist, who suspected a malignant parotid gland tumor with skull base invasion, and thus, referred the patient to our department. The patient had no medical history to note. He did not present with any cranial nerve symptoms, and pharyngolaryngeal endoscopy did not reveal any obvious structural abnormalities. Contrast-enhanced magnetic resonance imaging (MRI) demonstrated a pale, somewhat poorly defined mass in the right parapharyngeal space. Contiguity with the parotid gland was not evident. While the tumor had partially invaded the skull base, there was no obvious extension into the prevertebral muscles or neck (Figure 1). Moreover, there were no apparent findings indicative of metastases to multiple sites, such as the cervical lymph nodes.

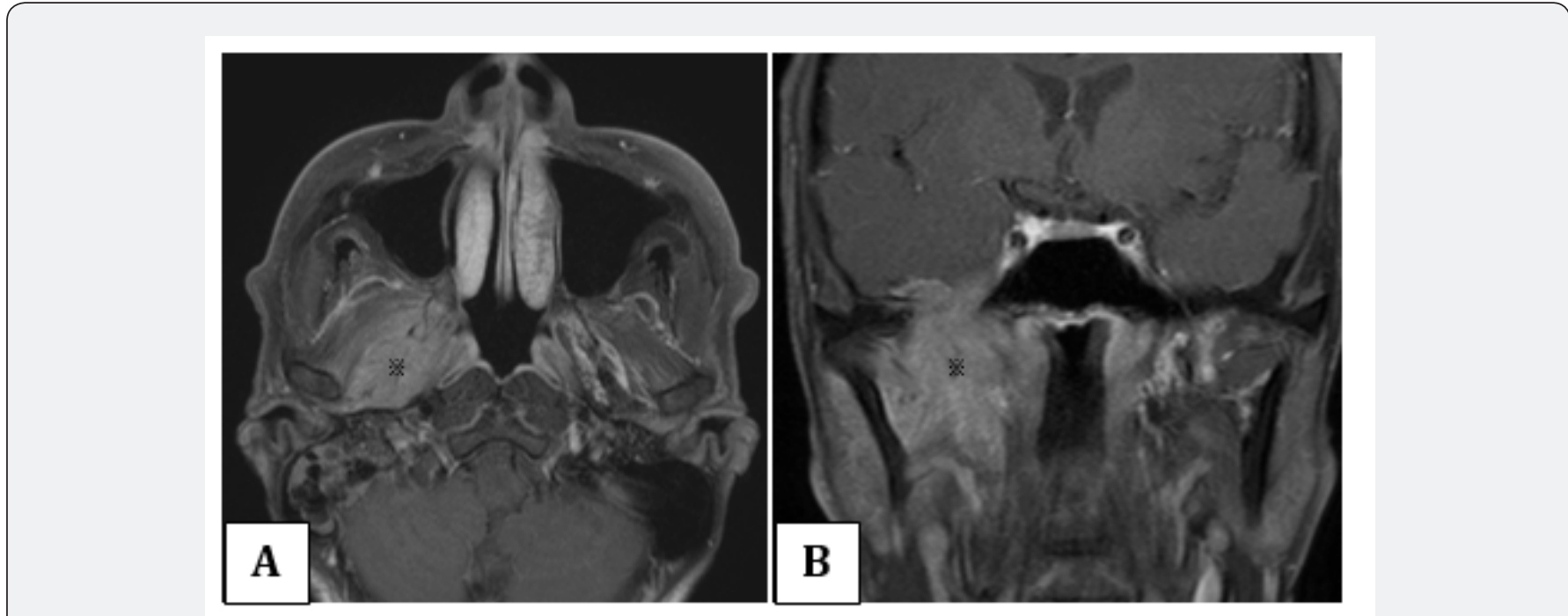

Figure 1: Enhanced MRI before biopsy in Case 1. The tumor $(※)$, whose border was moderately indistinct with the faint contrasting effect, was found in the right parapharyngeal space. It partially extended to the skull base, although it did not extend to the prevertebral muscle and neck.

The patient underwent a biopsy under general anesthesia. We performed the biopsy from the deep parotid lobe using the transcervical approach, and intraoperative pathological diagnosis with a frozen specimen showing chronic inflammation of the parotid tissue. The operation was completed by adding biopsies from the tissues around the parapharyngeal space using the transparotid approach. In the final pathological diagnosis, hematoxylin, and eosin (H\&E) staining revealed diffuse hyperplasia of large lymphoma cells that were round or oval-shaped, and had constricted nuclei, leading to a diagnosis of lymphoma. More specifically, based on immunostaining, the diagnosis was determined to be diffuse large B cell lymphoma (Figure 2).

Following diagnosis, the patient underwent six cycles of rituximab plus cyclophosphamide, doxorubicin, vincristine, and prednisone (R-CHOP) therapy at the referring hospital. On further imaging (CT, MRI, positron emission tomography [PET]), the tumor was found to have disappeared. Following the treatment, the patient has remained cancer-free for two years and nine months.

\section{Case 2}

A 60-year-old man developed left neck pain. One month after the onset of symptoms, he visited his otorhinolaryngologist who suspected a left nasopharyngeal tumor. After two biopsies failed to yield a diagnosis, the patient was referred to our department. Nasopharyngeal endoscopy revealed a protrusion in the left nasopharynx, although the nasal mucosal surface was normal (Figure 3A). MRI showed a poorly defined mass with contrast effect in the left parapharyngeal space. It was not evidently contiguous with the parotid gland and had displaced the nasopharynx (Figure 3B). Furthermore, on performing fluorodeoxyglucose (FDG)-PET, an intense uptake of FDG was observed in the same site (Figure 3C).

The patient underwent biopsy of the left nasopharynx under general anesthesia. We approached the lateral wall of the 


\section{Global Journal of Otolaryngology}

nasopharynx via the nasal cavity with an endoscope, made an incision in the mucosa, and biopsied the deep tissue there. Small to medium-sized lymphocytes were growing diffusely, peripheral to the submucosal glands; however, these lymphocytes did not demonstrate evident atypia. Consequently, the histological diagnosis was chronic inflammation (Figure 4A). Nasopharyngeal endoscopy four weeks after the biopsy revealed a marked protrusion in the left lateral nasopharyngeal wall (Figure 5A). On MRI, the tumor had grown markedly along the pharyngeal space not only in the direction of the nasopharyngeal cavity but also inferiorly towards the neck and superiorly towards the skull base (Figure 5B).

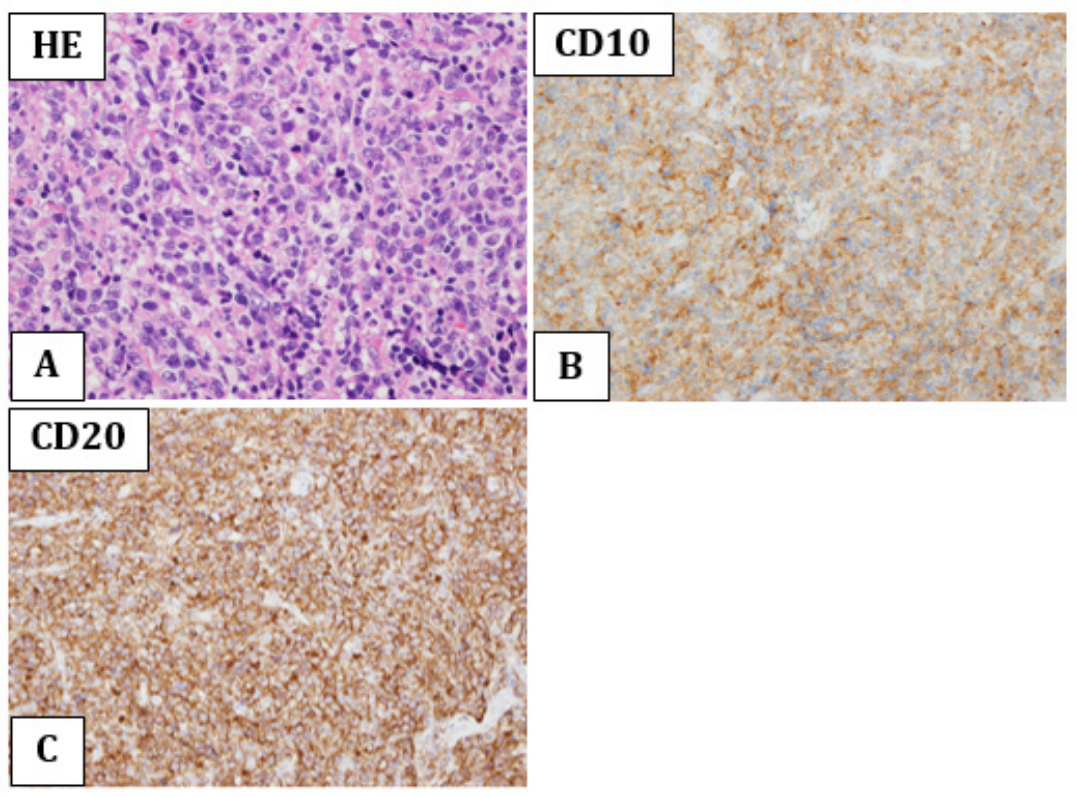

Figure 2: Pathological examination (H\&E staining and immunostaining) in Case 1. H\&E staining revealed diffuse hyperplasia of large lymphoma cells that were round or oval-shaped and had constricted nuclei (A), leading to a diagnosis of malignant lymphoma. The diagnosis was determined to be diffuse large B cell lymphoma by immunostaining of CD10 and CD20 (B and C).

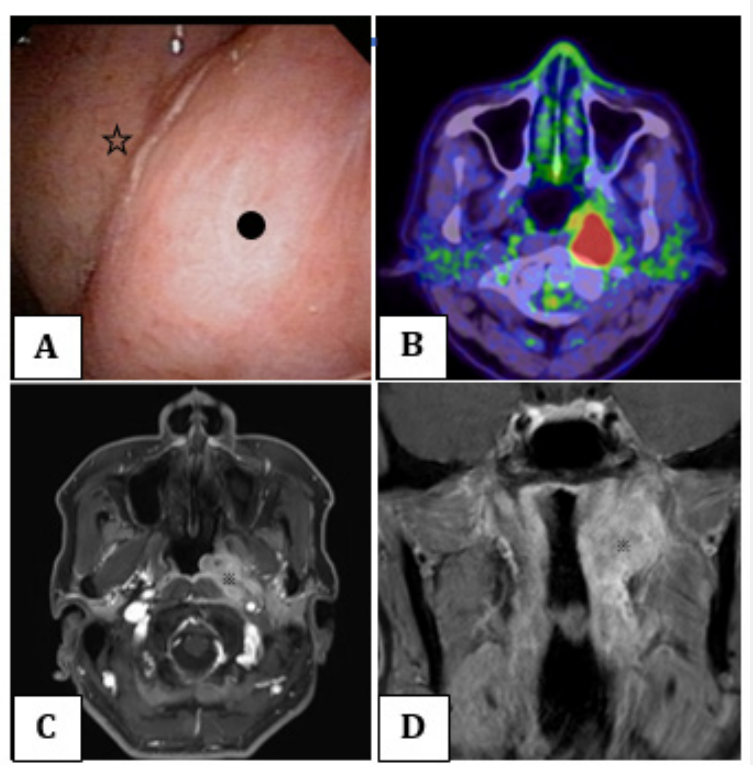

Figure 3: Images before first biopsy in Case 2. A) Nasopharyngeal fiber, Tubal elevation $(\bullet)$ was projected by the tumor in the left nasopharynx, although the mucous membrane was normal. $z$ : pharyngeal recess; B) FDG-PET There was strong FDG accumulation in the left nasopharynx. C and D. Enhanced MRI. We confirmed that the tumor's (※) border was moderately indistinct with the contrasting effect in the left parapharyngeal space. The tumor displaced the nasopharynx, and the continuity with the parotid gland was not apparent. 


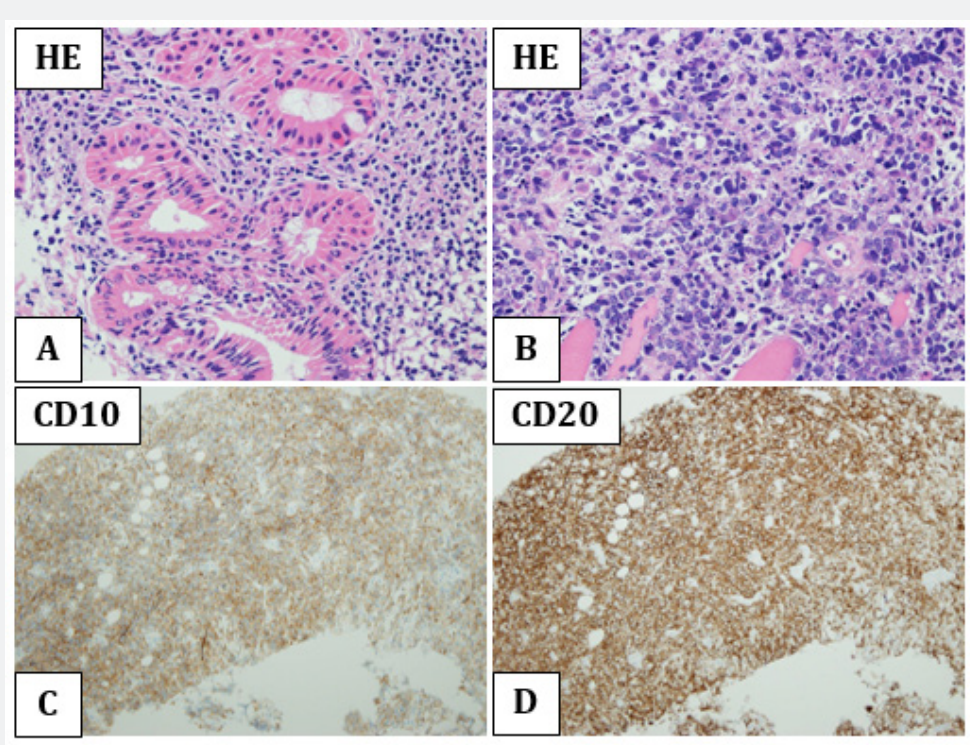

Figure 4: Pathological examination (H\&E staining and immunostaining) in Case 2; A) First biopsy H\&E staining exhibited small to mediumsized lymphocytes growing diffusely, peripherally to the submucosal glands. Without evident atypical nuclei in these lymphocytes, the histological diagnosis was chronic inflammation. B, C, and D. Second biopsy H\&E staining revealed diffuse hyperplasia of large lymphoma cells which were round or oval-shaped and had constricted nuclei (B). The combination of H\&E staining results with those of immunostaining for CD10 and CD20 (C and D) yielded a diagnosis of diffuse large B cell lymphoma.

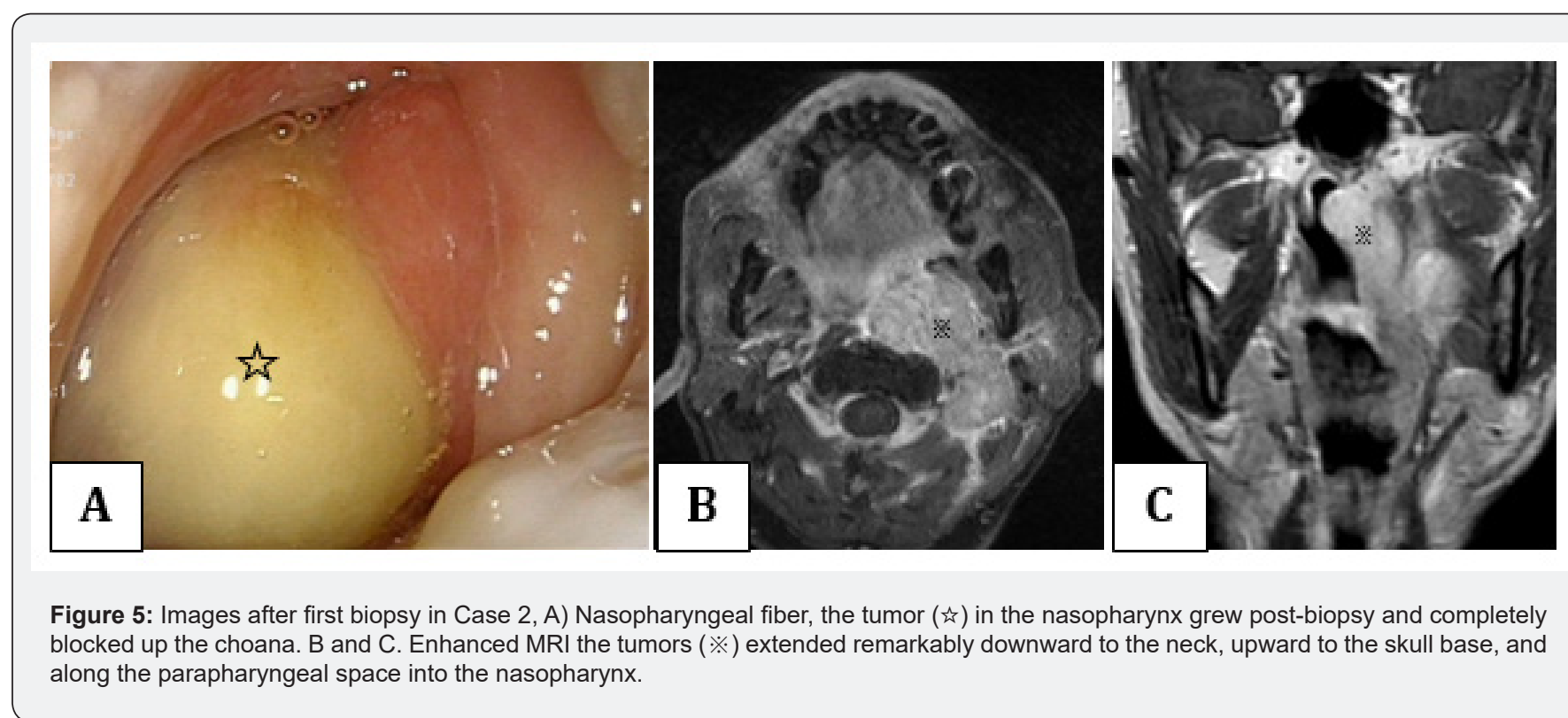

Although the patient complained of intense neck pain and headache, cranial nerve symptoms were not evident at this point. After biopsy, the tumor grew rapidly, strongly suggesting malignancy. Core needle biopsy (CNB) was performed from below the left ear. Like Case 1, H\&E staining exhibited diffuse hyperplasia of large lymphoma cells that were round or oval-shaped and had constricted nuclei; combination of $\mathrm{H} \& \mathrm{E}$ staining results with immunostaining results yielded a diagnosis of diffuse large B cell lymphoma (Figure 4B). Following two cycles of R-CHOP therapy at our hospital, the tumor appeared to have shrunk dramatically on MRI, and the patient achieved complete response (Figure 6). Subsequently, the patient underwent one additional cycle of R-CHOP therapy followed by a 40 gray (Gy) dosage of radiotherapy. The patient survived and has been tumor-free for four months after treatment. Moreover, the earlier complaints of intense neck pain and headache disappeared following one course of R-CHOP therapy. 


\section{Global Journal of Otolaryngology}
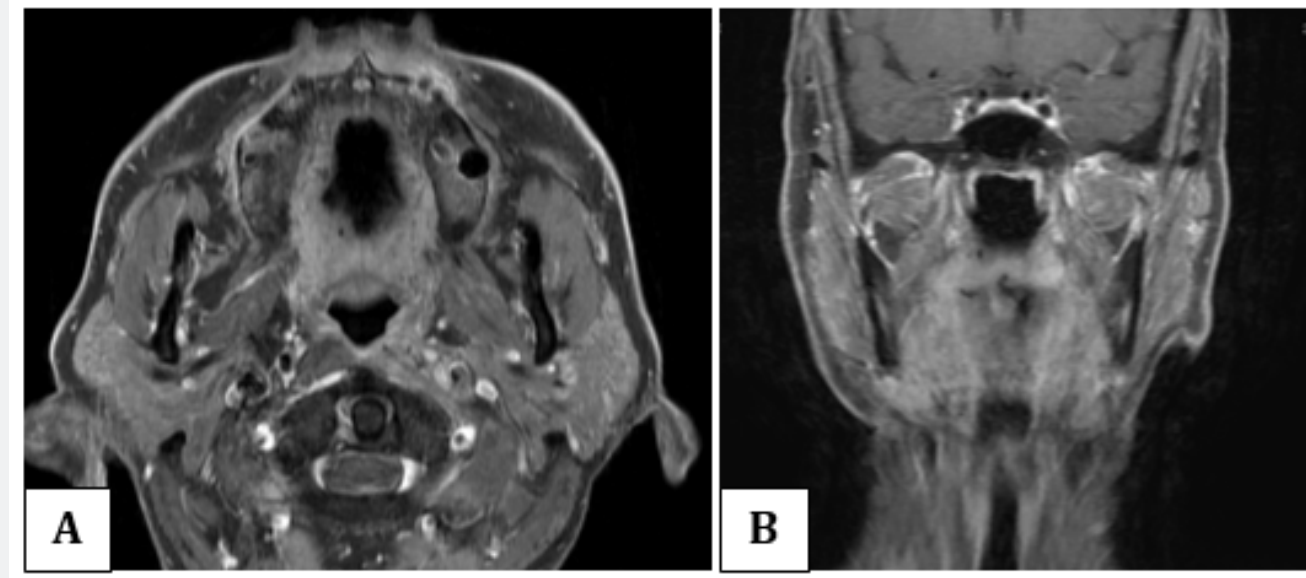

Figure 6: Enhanced MRI after treatment in Case 2. After two courses of R-CHOP treatment, the tumor reduced remarkably and had a diagnosis of complete response.

\section{Discussion}

Parapharyngeal space tumors present as diverse histological tissue types [6], hindering preoperative histological diagnosis; therefore, differentiation with MRI or CT is crucial [7]. The parapharyngeal space is broadly divided by the styloid process into an anterior pre-styloid compartment and a posterior poststyloid compartment. In general, we differentiate parapharyngeal tumors from structures existing in each area. In pre-styloid compartment, parotid gland tumor is most common; there are lipoma, lymphoma, and their malignant tumors elsewhere. On the other hand, in post-styloid compartment, neurogenic tumor is most common; there are paraganglioma, connective tissue tumor, carotid aneurysm, lymphoma, and their malignant tumors elsewhere [1]. Regarding neurogenetic tumors, originating nerves can be differentiated from the positional relationship between the internal and external carotid arteries and the internal jugular vein [8].

The two cases in this study demonstrated diffuse invasive masses that were suspected malignant tumors and were in the pre-styloid compartment. However, the tumors differed from typical imaging findings of parotid gland malignant tumors as the continuity with the parotid gland was unclear. Moreover, these parapharyngeal space tumors present image views unlike the nasopharyngeal carcinoma considering the invasive style to surrounding tissues [9]. As the tumors were in the prestyloid compartment, we suspected liposarcoma and malignant lymphoma. Moreover, as liposarcoma showed high signal density areas on T1-weight images of MRI [10], it was thought that the tumor was negative in these two cases. From the past report, we eventually suspected malignant lymphoma that was extra nodal, which diffusely increased along the parapharyngeal space [11].

Malignant lymphoma is broadly divided into nodal lymphoma and extranodal lymphoma. While the cervical lymphadenopathy that otorhinolaryngologists encounter is often depicted in imaging as a nodal lymphoma, extranodal lymphoma is rare in the head and neck region [12]. Both cases of parapharyngeal space malignant lymphomas in the present study were morphologically extranodal lymphomas and demonstrated growth along the parapharyngeal space $[11,13]$. Case 2 was considered distinctive in that the tumor originated in the parapharyngeal space, extended extensively along the space towards the neck and skull base, and caused the patient intense pain despite the absence of symptoms associated with the involvement of cranial nerves. One of the characteristic symptoms in suspected malignant lymphoma cases is a strong pain, due to the tumor enlargement. It was not associated with neurologic symptoms for these two cases, but there was the case of parapharyngeal malignant lymphoma with the cranial nerve paralysis in the past report [11].

It is difficult to perform a biopsy, and to approach parapharyngeal tumors. In Case 1, where the tumor extended from the deep parotid lobe to the skull base, we performed an open biopsy utilizing transcervical and trans parotid approach. Moreover, in Case 2, where the tumor extended inferiorly from the lateral nasopharynx, we performed an ultrasound guided CNB from transcervical approach. While CT-guided fine-needle aspiration cytology is reported to be effective for preoperative diagnosis and treatment [14], cytology is obviously ill-suited for diagnosing parotid gland tumors, neurologic tumors, and nonepithelial malignancies. Therefore, we believe that a preoperative CNB or open biopsy is necessary in parapharyngeal space tumor. We choose the method of the following approaches based on the property of tumor and the surrounding structure: transcervical, trans parotid, transoral, and trans nasopharynx. In the case of post-styloid compartment, it is necessary to take care of cranial neuro paralysis and bleeding at the biopsy because there is a highly probability to be neurogenic tumor or angiogenic tumor. 
In case 2, it is important to discuss why the tumor increased dramatically after the first biopsy. We have two hypotheses to explain the increasing size after the first biopsy. The first hypothesis is that inflammatory lymphatic growth was present around DLBCL. It is not rare that a biopsy or infection exacerbates malignant lymphoma with chronic inflammation. The second hypothesis is that it was a follicular lymphoma at first biopsy, pathological diagnosis is thought to be difficult, and the biopsy induced to transform histologically into aggressive DLBCL [15]. In conclusion, diagnosis of extra nodal malignant lymphoma in the parapharyngeal space using images from CT and MRI is possible, to some extent, by visualizing whether the tumor has increased along the space. However, pathological diagnosis by CNB or open biopsy is difficult, and several biopsies in diverse ways might be required. Therefore, thorough knowledge of the anatomy of the parapharyngeal space and the surrounding structures is necessary to perform biopsies with various approaches.

\section{Conclusion}

We describe two cases of malignant lymphomas originating from the parapharyngeal space. Biopsy-based pathological diagnosis of pharyngeal space tumors is often difficult; therefore, diagnostic imaging with CT or MRI and a strategy for biopsy are crucial. These cases suggest that masses that grow diffusely along the parapharyngeal space towards the neck and skull base and cause the patient to complain of intense pain, are highly likely to be malignant lymphomas.

\section{Acknowledgment}

The authors would like to thank N. Oshima, MD/PhD, for her advice on this article from the viewpoint of a blood physician.

\section{References}

1. Kuet ML, Kasbekar AV, Masterson L, Jani P (2015) Management of tumors arising from the parapharyngeal space: A systematic review of 1,293 cases reported over 25 years. Laryngoscope 125(6): 1372-1381.

2. Lien KH, Young CK, Chin SC, Liao CT, Huang SF (2019) Parapharyngeal space tumors: a serial case study. J Int Med Res 47(8): 4004-4013.

3. Papadogeorgakis N, Petsinis V, Goutzanis L, Kostakis G, Alexandridis C
(2010) Parapharyngeal space tumors: surgical approaches in a series of 13 cases. Int J Oral Maxillofac Surg 39(3): 243-250.

4. Chan JY, Tsang RK, Eisele DW, Richmon JD (2015) Transoral robotic surgery of the parapharyngeal space: a case series and systematic review. Head Neck 37(2): 293-298.

5. Pilolli F, Giordano L, Galli A, Bussi M (2016) Parapharyngeal space tumours: video-assisted minimally invasive transcervical approach. Acta Otorhinolaryngol Ital 36(4): 259-264.

6. Locketz GD, Horowitz G, Abu Ghanem S, Wasserzug O, Abergel A, et al. (2016) Histopathologic classification of parapharyngeal space tumors: a case series and review of the literature. Eur Arch Otorhinolaryngol 273(3): 727-734.

7. Liu XW, Wang L, Li H, Zhang R, Geng ZJ, et al. (2014) A modified method for locating parapharyngeal space neoplasms on magnetic resonance images: implications for differential diagnosis. Chin J Cancer 33(10): 511-520.

8. Shirakura S, Tsunoda A, Akita K, Sumi T, Suzuki M, et al. (2010) Parapharyngeal space tumors: anatomical and image analysis findings. Auris Nasus Larynx 37(5): 621-625.

9. Liu XW, Xie CM, Mo YX, Zhang R, Li H, et al. (2012) Magnetic resonance imaging features of nasopharyngeal carcinoma and nasopharyngeal non-Hodgkin's lymphoma: are there differences? Eur J Radiol 81(6): 1146-1154.

10. Kikuchi N, Nakashima T, Fukushima J, Nariyama K, Komune S (2015) Well-differentiated liposarcoma arising in the parapharyngeal space: a case report and review of the literature. J Laryngol Otol 129(2): S8690.

11. Tülin Kayhan F, Ozkul N (1999) Case report: extranodal non-Hodgkin's lymphoma of the parapharyngeal space. Auris Nasus Larynx 26(2): 201-205.

12. Picard A, Cardinne C, Denoux Y, Wagner I, Chabolle F, et al. (2015) Extranodal lymphoma of the head and neck: a 67-case series. Eur Ann Otorhinolaryngol Head Neck Dis 132(2): 71-75.

13. Qureshi SS, Shet TM, Nagarajan G, Dcruz AK (2006) Extranodal nonHodgkin's lymphoma of the parapharyngeal space. Indian J Med Sci 60(4): 159-161.

14. Oliai BR, Sheth S, Burroughs FH, Ali SZ (2005) "Parapharyngeal space" tumors: a cytopathological study of 24 cases on fine-needle aspiration. Diagn Cytopathol 32(1): 11-15.

15. González Rincón J, Méndez M, Gómez S, García JF, Martín P, et al. (2019) Unraveling transformation of follicular lymphoma to diffuse large B-cell lymphoma. PLoS One 14(2): e0212813.

\begin{tabular}{l} 
Your next submission with Juniper Publishers \\
will reach you the below assets \\
- Quality Editorial service \\
- Swift Peer Review \\
- Reprints availability \\
- E-prints Service \\
- Manuscript Podcast for convenient understanding \\
- Global attainment for your research \\
- Manuscript accessibility in different formats \\
( Pdf, E-pub, Full Text, Audio) \\
- Unceasing customer service \\
Track the below URL for one-step submission \\
https://juniperpublishers.com/online-submission.php \\
\hline
\end{tabular}

